\title{
CONVOLUTION OPERATORS ON G-HOLOMORPHIC FUNCTIONS IN INFINITE DIMENSIONS
}

\author{
BY \\ PHILIP J. BOLAND AND SEÁN DINEEN
}

\begin{abstract}
For a complex vector space $E$, let $H_{G}(E)$ denote the space of $G$ (Gateaux)-holomorphic functions on $E$ ( $f: E \rightarrow C$ is $G$-holomorphic if the restriction of $f$ to every finite dimensional subspace of $E$ is holomorphic in the usual sense). The most natural topology on $H_{G}(E)$ is that of uniform convergence on finite dimensional compact subsets of $E$. A convolution operator $A$ on $H_{G}(E)$ is a continuous linear mapping $A: H_{G}(E) \rightarrow H_{G}(E)$ such that $A$ commutes with translations. The concept of a convolution operator generalizes that of a differential operator with constant coefficients. We prove that if $A$ is a convolution operator on $H_{G}(E)$, then the kernel of $A$ is the closed linear span of the exponential polynomials contained in the kernel. In addition, we show that any nonzero convolution operator on $H_{G}(E)$ is a surjective mapping.
\end{abstract}

Introduction. It is the object of this paper to construct weighted subspaces of $H_{G}(E)$ and to study convolution operators on them. $H_{G}(E)$ will denote the complex vector space of $G$ (Gateaux)-holomorphic functions on the complex vector space $E$. The most natural topology on $H_{G}(E)$ is that of uniform convergence on finite dimensional compact subsets of $E$. For each $n, P_{a}\left({ }^{n} E\right) \subseteq H_{G}(E)$ where $P_{a}\left({ }^{n} E\right)$ is the space of $n$-homogeneous polynomials on $E$. $P_{a}^{\prime}\left({ }^{n} E\right)$ is naturally isomorphic with $P\left({ }^{n} E^{*}\right)$, the space of $n$-homogeneous polynomials which are continuous on the algebraic dual of $E$ with respect to the weak topology $\sigma\left(E^{*}, E\right)$. $\mathcal{K}$ will denote a family of nonnegative functions on $E$ with finite dimensional support satisfying certain conditions. It is these families $\mathcal{K}$ which act as weights. Given $E$ and $\mathcal{K}$ defined on $E, H_{G}(E, \mathcal{K})=\left\{f: f \in H_{G}(E)\right.$ and $|f(z) k(z)|$ is bounded on $E$ for each $k \in \mathcal{K}\}$. Dual to $\mathcal{K}$, one defines the family $\mathcal{K}^{*}=\left\{k^{*}: k^{*}\right.$ maps $E \rightarrow$ $[0,+\infty), k^{*}$ has finite dimensional support and its restriction to every finite dimensional subspace is upper semicontinuous, and $k(z) k^{*}(\phi) e^{\phi(z)}$ is bounded on $E \times E^{*}$ for each $\left.k \in \mathcal{K}\right\}$. Also $H\left(E^{*}, \mathcal{K}^{*}\right)=\left\{f: f \in H\left(E^{*}\right),\left|f(\phi) k^{*}(\phi)\right|\right.$ is bounded on $E^{*}$ for every $\left.k^{*} \in \mathcal{K}^{*}\right\}$. For certain $\mathcal{K}$, if $H_{G}(E, \mathcal{K})$ is endowed with the

Received by the editors November 28, 1972 and, in revised form, April 25, 1973. AMS (MOS) subject classifications (1970). Primary 46E10, 47F05; Secondary 46L20, $32 \mathrm{~A} 15,41 \mathrm{~A} 30$.

Key words and phrases. Convolution operator, $G$ (Gateaux)-holomorphic function, weighted space of $G$ (Gateaux)-holomorphic functions, exponential-polynomial, Borel transform. 
topology defined by the family of seminorms

$$
||_{k}: f \rightarrow \sup _{z \in E}|f(z) k(z)| \quad \text { for each } k \in K
$$

then $H_{G}^{\prime}(E, K)$ can via the Borel transform be identified with $H\left(E^{*}, K^{*}\right)$.

It can be shown that the convolution operators on $H_{G}(E, K)$ are algebraically isomorphic to $H_{G}^{\prime}(E, \mathcal{K})$. For certain $K, H\left(E^{*}, K^{*}\right)$ satisfies the standard division theorem (if $F_{1}, F_{2} \in H\left(E^{*}, K^{*}\right.$ ) and $F_{1} / F_{2} \in H\left(E^{*}\right)$, then $F_{1} / F_{2} \in H\left(E^{*}, K^{*}\right)$ ). From this, together with the Borel transform identification, it follows that the kernel of any convolution operator is the dense linear span of the exponential polynomials contained therein. Furthermore, every nonzero convolution operator on $H_{G}(E, K)$ is a surjective mapping.

For appropriately chosen $K$, one obtains the following examples:

1. $H_{G}(E)$,

2. the $G$-holomorphic functions of finite order,

3. the $G$-holomorphic functions of exponential type.

The aforementioned results on convolution operators are valid for each of these examples.

An important application results from the fact that if $\Sigma_{N} \mathrm{C}$ is endowed with the locally convex inductive limit topology, then $H\left(\Sigma_{N} \mathrm{C}\right)=H_{G}\left(\Sigma_{N} \mathrm{C}\right)$. Hence the results concerning convolution operators hold also for $H\left(\Sigma_{N} \mathrm{C}\right)$.

Results similar to these were proved by B. Malgrange [6] and B. A. Taylor [8] in the finite dimensional case, and by $C$. Gupta [5] in the infinite dimensional case.

The notation used herein will principally be that of Gupta [5], Nachbin [7], and Taylor [8].

1. Polynomials. For a given complex vector space $E, H_{G}(E)$ will denote the space of all $\mathrm{C}$-valued functions on $E$ whose finite dimensional restrictions are holomorphic functions. That is, $H_{G}(E)$ is the space of $G$ (Gateaux)-holomorphic functions on $E$. If a locally convex topology is associated with $E$, then $H(E)$ will denote the space of $G$-holomorphic functions on $E$ which are continuous with respect to the given topology. $H_{G}(E)$ is a complete locally convex topological vector space when endowed with the topology of uniform convergence on finite dimensional compact subsets of $E . P_{a}\left({ }^{n} E\right)$ for each $n=0,1, \cdots$ is the space of $n$-homogeneous polynomials on $E$. Clearly $P_{a}\left({ }^{n} E\right) \subseteq H_{G}(E)$. Moreover, given $f \in H_{G}(E)$, there exist unique polynomials $\hat{d}^{n} f(0) / n ! \in P_{a}\left({ }^{n} E\right), n=0,1, \cdots$, such that $f(z)=\sum_{n=0}^{\infty} \hat{d}^{n} f(0)(z) / n$ ! for each $z \in E$. $E^{*}$ is the algebraic dual of $E$ endowed with the weak topology $\sigma\left(E^{*}, E\right)$, and $P\left({ }^{n} E^{*}\right)$ is the space of continuous $n$-homogeneous polynomials on $E^{*}$. 
Proposition 1. $P_{a}^{\prime}\left({ }^{n} E\right)$ and $P\left({ }^{n} E^{*}\right)$ are algebraically isomorphic where $P_{a}^{\prime}\left({ }^{n} E\right)$ is the continuous dual of $P_{a}\left({ }^{n} E\right)$, and $P_{a}\left({ }^{n} E\right)$ is endowed with the compact open topology for finite dimensional compact sets.

Proof. Define $\beta: P_{a}^{\prime}\left({ }^{n} E\right) \rightarrow P\left({ }^{n} E^{*}\right)$ such that if $T \in P_{a}^{\prime}\left({ }^{n} E\right)$ and $\phi \in E^{*}$, then $\beta T(\phi)=T\left(\phi^{n}\right)$. Clearly $\beta$ is a well defined linear mapping into $P\left({ }^{n} E^{*}\right), \beta$ is $1-1$ since the linear span of $\left\{\phi^{n}: \phi \in E^{*}\right\}$ is dense in $P_{a}\left({ }^{n} E\right)$.

Now if $p^{\prime} \in P\left({ }^{n} E^{*}\right)$, there exists a finite dimensional subspace $F^{*}$ of $E^{*}$ and a continuous projection $\pi$ of $E^{*}$ onto $F^{*}$ such that $p^{\prime}(\phi)=p^{\prime}(\pi \phi)$ for each $\phi \in E^{*}$. (See, for example, Barroso [1].) Using this together with the fact that $\beta$ is surjective when $E$ is finite dimensional, one may find a $T_{p^{\prime}} \in P^{\prime}\left({ }^{n} E^{*}\right)$ such that $\beta T_{p^{\prime}}=p^{\prime}$. (See Gupta [5, p. 59].)

2. Weighted spaces of $G$-holomorphic functions.

Definition 1. For a given complex vector space $E, K$ will denote any family of nonnegative functions on $E$ with the following properties ( $K$ will be referred to as a family of weights on $E$ ):

(i) For each $k \in \mathcal{K}$, there exists a finite dimensional subspace $F$ of $E$ such that $k$ is zero outside of $F$, and $k$ restricted to $F$ is upper semicontinuous.

(ii) If $k_{1}, k_{2} \in \mathcal{K}$ and $C>0$, then $C k_{1} \in \mathcal{K}$ and $\max \left(k_{1}, k_{2}\right) \in \mathcal{K}$. Also if $0 \leq k_{1} \leq k_{2}, k_{2} \in \mathcal{K}$ and $k_{1}$ satisfies (i), then $k_{1} \in \mathcal{K}$. If $k$ is the characteristic function of a finite dimensional compact set, then $k \in \mathcal{K}$.

(iii) If $k \in \mathcal{K}$ and $\epsilon>0$, then $z \in E \rightarrow k(\epsilon z)$ belongs to $\mathcal{K}$.

(iv) $\mathcal{K}$ is radial. That is for $k \in \mathcal{K}$, there exist $k_{1} \in \mathcal{K}$ and a finite dimensional subspace $F$ of $E$ with the following properties: (a) $k \leq k_{1}$ on $E$, (b) $k_{1}$ is 0 outside $F$, and (c) there exists a norm ||$_{F}$ on $F$ such that if $|w|_{F}=|z|_{F}$, then $k_{1}(w)=k_{1}(z)$.

(v) If $\phi \in E^{*}$, then $e^{\phi(z)} k(z)$ is bounded on $E$.

(vi) If $k_{1}, k_{2} \in \mathcal{K}$, then so does $k_{1} * k_{2}$ where $k_{1} * k_{2}(w)=$ $\sup _{z \in E} k_{1}(z) k_{2}(w-z)$.

(vii) Let $K_{F}$ denote the restrictions of $\mathcal{K}$ to $F$, where $F$ is a finite dimensional subspace. Then $K_{F}$ is hypoconvex. (Suppose $F$ is a finite dimensional subspace of $E,||_{F}$ is a norm on $F$, and $R(+)$ is the set of nonnegative reals. Let $\lambda(r)$ be a log convex function on $R(+)$ which is continuously differentiable and nondecreasing such that $k\left(|z|_{F}\right) e^{\lambda\left(|z|_{F}\right)}$ is bounded on $F$ for all $k \in K_{F}$. Then $\mathcal{K}_{F}$ is said to be hypoconvex if there exists a convex function $\lambda_{1}$ on $R(+)$ such that $\lambda_{1}(r) \geq \lambda(r)$ for all $r \in R(+)$ and $k\left(|z|_{F}\right) e^{\lambda_{1}(|z| F)}$ is bounded on $F$ for all $k \in K_{F}$. See Taylor [8, p. 447] for a discussion of hypoconvexity.)

We now define the spaces $H_{G}(E, K)$ which are considered in this paper. Several explicit examples are given at the end of this section. 
Definition 2. Given a complex vector space $E$ and a family $\mathcal{K}$ of weights on $E$, let

$$
H_{G}(E, K)=\left\{f: f \in H_{G}(E) \text { and } \sup _{z \in E}|f(z) k(z)|<+\infty \text { for all } k \in K\right\} \text {. }
$$

For each $k \in \mathcal{K}$, let ||$_{k}$ be the seminorm such that

$$
|f|_{k}=\sup _{z \in E}|f(z) k(z)| \text {. }
$$

$H_{G}(E, K)$ endowed with the topology generated by all seminorms of the form $\mid I_{k}$ is a complete locally convex topological vector space. The conditions (i)$(v)$ in the definition of a family of weights $\mathcal{K}$ on $E$ are quite natural and ensure that $H_{G}(E, K)$ has some reasonable basic properties. Let $f$ be a $G$-holomorphic function on $E, F$ a finite dimensional subspace of $E$, and ||$_{F}$ some norm on $F$. For each $r>0$ define $M\left(r,\left.f\right|_{F}\right)=\left.\sup _{|z|}\right|_{F=r}|f(z)|_{\text {. Conditions (i) and (iv) on } K}$ show that whether or not $f \in H_{G}(E, K)$ is determined by the growth of the functions $M\left(r,\left.f\right|_{F}\right)$. Condition (iii) on $K$ implies the following: Suppose $f \in H_{G}(E, K)$, $g \in H_{G}(E)$, and that for every finite dimensional subspace $F$ and norm ||$_{F}$ on $F$ there exists a constant $A$ (depending on $F$ and ||$_{F}$ ) such that $M\left(r,\left.g\right|_{F}\right) \leq$ $M\left(A r,\left.f\right|_{F}\right)$ for all $r>0$-then $g \in H_{G}(E, K)$. Condition $(v)$ on $K$ ensures that $H_{G}(E, K)$ contains all $G$-holomorphic functions which are of exponential type on every finite dimensional subspace of $E$ (and hence in particular every polynomial).

Condition (vi) on $\mathcal{K}$ guarantees that every convolution operator on $H_{G}(E, K)$ is of the form $T *$ (see Proposition 3), while condition (vii) is a condition sufficient to establish the isomorphism of Proposition 2.

The following lemmas describe some of the basic properties of the spaces $H_{G}(E, K)$.

Lemma 1. If $\phi \in E^{*}$, then $e^{\phi} \in H_{G}(E, K)$.

Proof. This follows from condition ( $v$ ) on the family $K$.

If $f \in H_{G}(E, K)$ and $a \in E$, then $r_{-a} f$ will denote the translate of $f$ by $a\left(r_{-a} f(z)=f(z+a)\right.$ for each $\left.z \in E\right)$.

Lemma 2. $H_{G}(E, \mathcal{K})$ is translation invariant for all $\mathcal{K}$. Tbat is, if $a \in E$ and $f \in H_{G}(E, K)$, then $r_{-a} f \in H_{G}(E, K)$.

Proof. This is a consequence of condition (iii) on the family $K$.

Lemma 3. $H_{G}(E, K)=\left\{f: f \in H_{G}(E)\right.$; and if $F$ is a finite dimensional subspace of $E,||_{F}$ a norm on $F$ and $k \in \mathcal{K}$, then

$$
\sup _{r>0} \sup _{|z|_{F}=r}\left|M\left(r,\left.f\right|_{F}\right) k(z)\right|<+\infty .
$$


Lemma 4. If $f \in H_{G}(E, K)$, then $\sum_{n=0}^{m} \hat{d}^{n} f(0) / n ! \rightarrow f$ in the topology of $H_{G}(E, K)$ as $m \rightarrow \infty$.

Proof. Let $k \in \mathbb{K}$. Without loss of generality $k$ is zero outside some finite dimensional subspace $F$ of $E$ and for some norm ||$_{F}$ on $F, k(w)=k(z)$ whenever $|w|_{F}=|z|_{F}$ (by conditions (i) and (iii) of Definition 1). Let $M\left(r,\left.f\right|_{F}\right)=$ $\sup |z|_{F}=r|f(z)|$. Since $k$ is bounded, it suffices to show that

Now

$$
\lim _{m \rightarrow+\infty} \sup _{|z|_{F} \geq 2}\left|k(z) \sum_{n=m}^{\infty} \frac{\partial^{n} f(0)}{n !}(z)\right|=0
$$

$$
\sup _{|z|_{F} \geq 2}\left|k(z) \sum_{n=m}^{\infty} \frac{\hat{d}^{n} f(0)}{n !}(z)\right| \leq \sup _{|z|_{F} \geq 2}\left|k(z) \sum_{n=m}^{\infty} \frac{M\left(2|z|_{F},\left.f\right|_{F}\right)|z|_{F}^{n}}{\left(2|z|_{F}\right)^{n}}\right|
$$

(by the Cauchy inequalities)

$$
\begin{aligned}
& \leq\left(\sum_{n=m}^{\infty} 1 / 2^{n}\right) \sup _{|z|_{F} \geq 2}\left|k(z) M\left(2|z|_{F},\left.f\right|_{F}\right)\right| \\
& \rightarrow 0 \text { as } m \rightarrow+\infty
\end{aligned}
$$

Following standard notation, $H_{G}^{\prime}(E, \mathcal{K})$ will denote the continuous dual of $H_{G}(E, \mathcal{K})$, i.e., the space of continuous linear forms on $H_{G}(E, \mathcal{K})$. If $T \in H_{G}^{\prime}(E, \mathcal{K})$, then $\hat{T}$, the Borel transform of $T$, is the function defined on $E^{*}$ by

$$
\hat{T}: \phi \in E^{*} \rightarrow T\left(e^{\phi}\right) \text {. }
$$

$\hat{T}$ is holomorphic on $E^{*}$ and in fact depends only on a finite number of variables (this follows from the fact that we are considering the weak topology on $E^{*}$-see, for example, Barroso [1]). One may easily show that the linear span of $\left\{e^{\phi}: \phi \in E^{*}\right\}$ is dense in $H_{G}(E, \mathcal{K})$. Hence the Borel transform ", where " : $T \in H_{G}^{\prime}(E, \mathcal{K}) \rightarrow$ $\hat{T} \in H\left(E^{*}\right)$, is an injective linear mapping.

Definition 3. For a given family $\mathcal{K}$ of weights on $E$, let $\mathcal{K}^{*}=\left\{k^{*} \mid k^{*}: E^{*} \rightarrow\right.$ $[0,+\infty)$, for some finite dimensional subspace $F^{*}$ of $E^{*}, k^{*}$ is zero outside of $F^{*}$ and upper semicontinuous on $F^{*}$, and if $k \in \mathcal{K}$ then $\sup _{z \in E, \phi \in E^{*}} \mid k(z) k^{*}(\phi) e^{\phi(z) \mid}$ $<+\infty\}$. Also let $H\left(E^{*}, K^{*}\right)=\left\{f: f \in H\left(E^{*}\right)\right.$, sup $\operatorname{suE}_{\phi}\left|f(\phi) k^{*}(\phi)\right|<+\infty$ for each $\left.k^{*} \in \mathcal{K}^{*}\right\}$.

$K^{*}$ satisfies conditions (i)-(v) of Definition 1 , although this fact will not be utilized.

Lemma 5. ' $\left(H_{G}^{\prime}(E, \mathcal{K})\right) \subseteq H\left(E^{*}, \mathcal{K}^{*}\right)$, i.e., if $T \in H_{G}^{\prime}(E, \mathcal{K})$, then $\hat{T} \in H\left(E^{*}, \mathcal{K}^{*}\right)$.

Proof. Since $\hat{T} \in H\left(E^{*}\right)$, it suffices to show that $\sup _{\phi \in E^{*}}\left|k^{*}(\phi) \hat{T}(\phi)\right|<+\infty$ 
for each $k^{*} \in \mathcal{K}^{*}$. Let $C>0$ and $k \in \mathcal{K}$ be such that $|T(f)| \leq C|f|_{k}$ for each $f \in H_{G}(E, K)$. If $k^{*} \in K^{*}$, then

$$
\left|k^{*}(\phi) \hat{T}(\phi)\right|=\left|k^{*}(\phi) T\left(e^{\phi}\right)\right| \leq C k^{*}(\phi) \sup _{z \in E}\left|k(z) e^{\phi(z)}\right| .
$$

Hence by definition of $\mathcal{K}^{*}$, it follows that $\sup _{\phi \in E^{*}}\left|k^{*}(\phi) \hat{T}(\phi)\right|<+\infty$.

Definition 4. (a) A family of weights $\mathcal{K}$ on $E$ is finitely of countable base (fcb) if $K_{F}$ is of countable base for each finite dimensional subspace $F$ of $E$ (i.e., for a given $F$, there exists an increasing sequence $\left(k_{n}\right)$ of elements of $K_{F}$ such that $k \in \mathcal{K}_{F} \Rightarrow k=O\left(k_{n}\right)$ for some $n$ ).

(b) $\mathcal{K}$ is finitely countably determined ( $\mathrm{fcd}$ ) if $\mathcal{K}_{F}$ is countably determined for each finite dimensional subspace $F$ of $E\left(K_{F}\right.$ is countably determined if there exist a norm ||$_{F}$ on $F$ and a sequence $\left(\psi_{n}\right)$ of continuous functions on $R(+)$, where $0<\psi_{1}(r)<\psi_{2}(r)<\cdots$ and $\psi_{n}$ is nondecreasing for each $n$, such that $K_{F}=\left\{k \mid k: F \rightarrow[0,+\infty), k(z) \psi_{n}\left(|z|_{F}\right)\right.$ is bounded on $F$ for each $\left.\left.n\right\}\right)$.

Proposition 2. If $\mathcal{K}$ is a family of weights on $E$ such that $\mathcal{K}$ is either fcb or $f c d$, then $H_{G}^{\prime}(E, K)$ and $H\left(E^{*}, K^{*}\right)$ are (algebraically) isomorphic via the Borel transform.

Proof. It suffices to show the Borel transform is a surjective mapping. Let $b \in H\left(E^{*}, K^{*}\right)$ be given. $b$ depends on a finite number of variables and hence there exist $\phi_{1}, \cdots, \phi_{m}$ linearly independent in $E^{*}$ such that $b$ is determined on the subspace $F^{*}$ generated by them. Let $F$ be a subspace of $E$ of dimension $m$ such that $F^{*}$ is the dual of $F$-i.e., let $F$ be generated by linearly independent $x_{1}, \cdots, x_{m} \in E$ such that $\phi_{j}\left(x_{i}\right)=\delta_{i j}$ for $i, j=1, \cdots, m$. The restriction of $b$ to $F^{*}$ is denoted $\left.b\right|_{F^{*}}$. Now $K_{F}$ is either of countable base or is countably determined. In either of these cases, it has been shown by Taylor [8] that $H^{\prime}\left(F, K_{F}\right)$ and $H\left(F^{*}, K_{F^{*}}\right)$ are isomorphic via the Borel transform. (Note $H\left(F, K_{F}\right)=$ $H_{G}\left(F, K_{F}\right)$.) Hence there exists $T_{F} \in H^{\prime}\left(F, \mathcal{K}_{F}\right)$ such that $\hat{T}_{F}=\left.b\right|_{F^{*}}$. Define $T: H_{G}(E, K) \rightarrow \mathrm{C}$ such that $T(f)=T_{F}\left(\left.f\right|_{F}\right)$. Clearly $T \in H_{G}^{\prime}(E, K)$. Now given $\phi \in E^{*}$, we can express $\phi=\phi_{1}+\phi_{2}$ in a unique manner where $\phi_{1} \in F^{*}$ and $\left.\phi_{2}\right|_{F}=0$. Hence for $\phi \in E^{*}$

$$
\hat{T}(\phi)=T\left(e^{\phi}\right)=T_{F}\left(\left.e^{\phi}\right|_{F}\right)=T_{F}\left(e^{\phi}\right)=\left.b\right|_{F^{*}}\left(\phi_{1}\right)=b\left(\phi_{1}+\phi_{2}\right)=b(\phi) .
$$

Therefore $\hat{T}=b$, completing the proof.

Some examples of weighted spaces are listed in the following chart. For each $K$, if $k \in \mathcal{K}$ then $k$ is assumed to have support in some finite dimensional subspace and to be upper semicontinuous there. 


\section{$\kappa$}

(1) $K_{c}=\{k: k$ has finite dimensional compact support\}

(2) $K_{e}=\left\{k: k e^{\phi}\right.$ is bounded for each $\left.\phi \in E^{*}\right\}$

(3) $K_{f O}=\left\{k: k(z) e^{|z|_{F}^{p}}\right.$ is bounded on $E$ for every $p>1$ and every ||$_{F}$ where ||$_{F}$ is a norm on the finite dimensional subspace $F\}$

(4) $K_{f O 1}=\{k$ : if $k$ has support in the finite dimensional $F$ and $\|\left.\right|_{F}$ is a norm on $F$, then $k(z)=O\left(e^{-|z|_{F}^{1+1 / n}}\right)$ for some $n\}$
$\underline{H_{G}(E, K)} \quad \underline{H\left(E^{*}, K^{*}\right)}$

$H_{G}(E) \quad \operatorname{Exp} E^{*}$

$\operatorname{Exp}_{G}(E)$ $H\left(E^{*}\right)$ $F O_{G}(E)$ FO1 $\left(E^{*}\right)$

$\mathrm{FOI}_{G}(E)$ $F O\left(E^{*}\right)$

$K_{c}$ and $K_{f O 1}$ are fcb while $K_{e}$ and $K_{f O}$ are fcd (Taylor [8, p. 452]). $\operatorname{Exp}_{G}(E)$ is the space of $G$-holomorphic functions on $E$ which are of exponential type on every finite dimensional subspace. $F O_{G}(E)$ and $F O 1_{G}(E)$ are the spaces of $G$. holomorphic functions on $E$ whose restrictions to finite dimensional subspaces are of finite order and of finite order $\leq 1$ respectively.

3. Convolution operators on $H_{G}(E, \mathcal{K})$. A convolution operator on $H_{G}(E, K)$ is a continuous linear mapping from $H_{G}(E, K)$ into itself which commutes with translations. $\mathcal{O}\left(H_{G}(E, K)\right)$ will denote the space of all such operators.

Proposition 3. $H_{G}^{\prime}(E, K)$ and $\mathcal{O}\left(H_{G}(E, K)\right)$ are (algebraically) isomorphic.

Proof. Define $\gamma: \mathcal{O}\left(H_{G}(E, K)\right) \rightarrow H_{G}^{\prime}(E, \mathcal{K})$ so that $\gamma(A)(f)=A f(0)$ where $A \in \mathcal{O}\left(H_{G}(E, K)\right)$ and $f \in H_{G}(E, K), y$ is a 1-1 linear mapping. For $T \in H_{G}^{\prime}(E, \mathcal{K})$ we may define $\gamma^{-1} T$ in the following way: $\gamma^{-1} T(f)(z)=T\left(r_{-z} f\right)$. For notational convenience, let $T * f(z)=T\left(\tau_{-z} f\right)$. Then $z \rightarrow T * f(z)$ is G-holomorphic. If $k \in \mathcal{K}$ and $C>0$ are such that $|T(g)| \leq C|g|_{k}$ for every $g \in H_{G}(E, \mathcal{K})$, then for $k_{1} \in \mathcal{K}$,

$$
\left|\gamma^{-1} T(f)(z) k_{1}(z)\right| \leq C\left|k_{1}(z)\right| \sup _{\omega \in E}|f(\omega+z) k(\omega)| .
$$

Hence $|T * f|_{k_{1}} \leq C|f|_{k_{1} * k}$. Therefore $T * f \in H_{G}(E, K)$ and also $f \rightarrow T * f$ is a 
continuous mapping. Since $\gamma^{-1} T$ is translation invariant, we have shown that $\gamma^{-1} T \in \Theta\left(H_{G}(E, K)\right)$. Now $\gamma^{-1} \circ \gamma$ and $\gamma^{\circ} \gamma^{-1}$ are the identities respectively on $\mathcal{O}\left(H_{G}(E, \mathcal{K})\right)$ and $H_{G}^{\prime}(E, \mathcal{K})$, and therefore $H_{G}^{\prime}(E, \mathcal{K})$ and $\Theta\left(H_{G}(E, \mathcal{K})\right.$ ) are (algebraically) isomorphic.

If $T_{1}$ and $T_{2} \in H_{G}^{\prime}(E, \mathcal{K})$, then there exist $A_{1}, A_{2} \in \Theta\left(H_{G}(E, K)\right)$ such that $\gamma\left(A_{i}\right)=T_{i}$ for $i=1,2$. Now $A_{1} \circ A_{2} \in \Theta\left(H_{G}(E, K)\right)$ and hence one defines $T_{1} * T_{2}=\gamma\left(A_{1} \circ A_{2}\right)$.

Definition 5. A family of weights $\mathcal{K}$ on $E$ satisfies condition $D(\mathcal{K}$ is $D)$ if $H\left(E^{*}, K^{*}\right)$ has a division theorem-i.e., if $b_{1}, b_{2} \in H\left(E^{*}, K^{*}\right)$ and $b_{1} / b_{2} \in H\left(E^{*}\right)$, then $b_{1} / b_{2} \in H\left(E^{*}, K^{*}\right)$.

Using growth estimates for a holomorphic function which is the quotient of two holomorphic functions, one may show that each of the $\mathcal{K}$ given as examples at the end of $\$ 2$ is $D$ (see Boland [3], and Taylor [8]). Sufficient conditions may be given to ensure that a $\mathcal{K}$ satisfying them is $D$ (Boland [3]).

Theorem 1. Let $\mathcal{K}$ be a family of weights on $E$ sucb that $\mathcal{K}$ is $D$ and $\mathcal{K}$ is either fcd or fcb. Then any convolution operator $A \in \Theta\left(H_{G}(E, K)\right)$ bas the property that its kernel is the closed linear span of the set $\left(p e^{\phi}: p \in P_{a}\left({ }^{n} E\right), \phi \in E^{*}\right.$, $\left.A\left(p e^{\phi}\right)=0\right\}$.

Proof. The method is that of Gupta [5]. If $A=0$, then the result is trivial so assume $A \neq 0$. There exists a $T \in H_{G}^{\prime}(E, \mathcal{K})$ such that $A=T *$. Let $S \in H_{G}^{\prime}(E, \mathcal{K})$ be such that $S\left(p e^{\phi}\right)=0$ whenever $T * p e^{\phi}=0$. Then it follows that $\hat{S} / \hat{T} \in H\left(E^{*}\right)$. Since $\mathcal{K}$ is $D$ and $H_{G}^{\prime}(E, \mathcal{K})$ and $H\left(E^{*}, K^{*}\right)$ are isomorphic via the Borel transform (Proposition 2), there exists $U \in H_{G}^{\prime}(E, K)$ such that $\hat{S}=\hat{U} \hat{T}$. Hence $S=$ $U * T \Rightarrow$ if $T * f=0$, then $S * f=(U * T) * f=U *(T * f)=0$. Therefore $T * f$ $=0$ implies that $S(f)=S * f(0)=0$ and hence the theorem follows with an application of the Hahn-Banach theorem.

Theorem 2. Let $\mathcal{K}$ be a family of weights on $E$ such that $\mathcal{K}$ is fcb or fcd. Then any nonzero convolution operator on $H_{G}(E, K)$ is a surjective mapping.

The following lemma, instrumental in the proof of Theorem 2, is established first.

Lemma 6. Suppose $A$ is a convolution operator on $H_{G}(E, K)$ wbicb is a surjective mapping. Then there exists a finite dimensional subspace $F$ of $E$ sucb that if $F_{1}$ is a subspace of $E$ containing $F$ then $A\left(H_{G}\left(E, F_{1}, K\right)\right)=H_{G}\left(E, F_{1}, K\right)$ where $H_{G}\left(E, F_{1}, \mathcal{K}\right)=\left\{f: f \in H_{G}(E, \mathcal{K})\right.$ and $\left.\left.f\right|_{F_{1}}=0\right\}$.

Proof. By Proposition 3 there is a $T \in H_{G}^{\prime}(E, K)$ such that $A=T *$. Let $k \in \mathcal{K}$ and $C>0$ be such that $|T(f)| \leq C|f|_{k}$ for all $f \in H_{G}(E, \mathcal{K})$. Choose $F$ 
to be the smallest subspace of $E$ outside of which $k$ is zero. $F$ is clearly finite dimensional. Let $F_{1}$ be a subspace containing $F$ and assume $f \in H_{G}\left(E, F_{1}, K\right)$. If $z \in F_{1}$, then

$$
|A f(z)|=|T * f(z)|=\left|T\left(\tau_{-z} f\right)\right| \leq C \sup _{\omega \in E}|k(w) f(z+w)|=0 .
$$

Therefore $A f \in H_{G}\left(E, F_{1}, K\right)$. It remains to show that for some $b \in H_{G}\left(E, F_{1}, K\right), A b=$ f. Now by assumption $A_{g}=f$ for some $g \in H_{G}(E, K)$. Let $\pi$ be a continuous projection of $E$ onto $F_{1}$, and define $\tilde{g}=g \circ \pi$. Note that $\tilde{g} \in H_{G}(E, K)$ and $g-\tilde{g} \in H_{G}\left(E, F_{1}, \mathcal{K}\right)$. Hence $T *(g-\tilde{g})=T * g-T * \tilde{g}=f-T * \tilde{g}$, and therefore $T * \tilde{g}(z)=T * \tilde{g}(\pi(z))=f(\pi(z))=0$ for all $z \in E$. But then $b=g-\tilde{g}$ is such that $b \in H_{G}\left(E, F_{1}, K\right)$ and $T * b=f-T * \tilde{g}=f$, which completes the proof of the lemma.

Proof of Theorem 2. The proof is by transfinite induction on the algebraic dimension of $E$. The theorem is known to be true for finite dimensional $E$ (Taylor [8]). Hence we assume the algebraic dimension of $E=\mu$ where $\mu$ is some infinite cardinal, and that the theorem holds for all $E$ with smaller algebraic dimension.

Suppose $A$ is a nonzero convolution operator on $H_{G}(E, K)$, and let $T, C, k$, and $F$ be as in Lemma 6 . There exists a nested family $\left(E_{a}\right)_{a \in I}$ of subspaces of $E$ such that each $E_{\alpha}$ is of algebraic dimension $<\mu$ and contains $F$, and $\bigcup_{a \in I} E_{a}$ $=E$. Fix a Hamel basis for $E$, say $\left(e_{j}\right)_{j \in J}$, such that each $E_{\alpha}$ is the subspace of $E$ generated by a specific subset of this basis. For each $E_{\alpha}$ let $E^{\alpha}$ be the algebraic complement of $E_{\alpha}$ generated by the elements in the Hamel basis which are not in $E_{\alpha}$.

Now for each a $\in I, T *$ naturally induces a nonzero convolution operator $T_{a} *$ on $H_{G}\left(E_{a},\left.K\right|_{E}\right)$. By the inductive hypothesis, $T_{a} *$ is a surjective mapping.

Let $f \in H_{G}(E, K)$ and consider a fixed $a \in I$. Let $f_{\alpha}$ be the restriction of $f$ to $E_{a}$. There exists $b_{1} \in H_{G}\left(E_{a},\left.K\right|_{E_{a}}\right)$ such that $T_{a} * b_{1}=f_{\alpha}$ Let $a_{1}>a$ and denote by $f_{a_{1} a_{1}}$ the natural extension of $f_{a}$ to $E_{a_{1}}$. More precisely, since $E_{a_{1}}=$ $E_{a} \oplus\left(E^{a} \cap E_{a_{1}}\right)$, every $z \in E_{a_{1}}$ can be uniquely expressed in the form $z=x+y$, $x \in E_{a}, y \in E^{a} \cap E_{a_{1}}$. Hence we define $f_{a_{1} a_{1}}$ on $E_{a_{1}}$ by $f_{a_{1} a_{1}}(z)=f_{a}(x)$. If $f_{a_{1}}=f f_{E_{a_{1}}}$, then $f_{a_{1}}$ and $f_{a_{1} a_{1}}$ agree on $E_{a}$ and therefore by Lemma 6 there exists $b_{2} \in H_{G}\left(E_{a_{1}}, E_{a},\left.K\right|_{E_{a_{1}}}\right)$ such that $T_{a_{1}} * b_{2}=f_{a_{1}}-f_{a_{1} a_{1}}$. Let $b_{1, a_{1}}$ denote the natural extension of $b_{1}$ to $E_{a_{1}}$. Then $T_{a_{1}} * b_{1, a_{1}}=f_{a_{1}, a_{1}}$. Hence

$$
\begin{aligned}
T_{a_{1}} *\left(b_{1, a_{1}}+b_{2}\right) & =T_{a_{1}} * b_{1, a_{1}}+T_{a_{1}} * b_{2} \\
& =f_{a_{1} a_{1}}+f_{a_{1}}-f_{a_{1} a_{1}}=f_{a_{1}} .
\end{aligned}
$$

Note moreover that $b_{1}, a_{1}+\left.b_{2}\right|_{E_{a}}=b_{1}$. Hence each solution of $T_{a} * b_{1}=f_{a}$ can 
be extended to $E_{a_{1}}$. Hence for a given $f \in H_{G}(E, K)$, a transfinite induction argument shows that for some $b \in H_{G}(E, K), T * b=f$.

Corollary 1. Let $\Sigma_{N} \mathbf{C}$ be a countable direct sum of $\mathbf{C}$ endowed with the locally convex inductive limit topology. If $H\left(\Sigma_{N} \mathrm{C}\right)$ is the space of bolomorphic functions on $\Sigma_{N} \mathbf{C}$ with the compact open topology, then the kernel of any convolution operator on $H\left(\Sigma_{N} \mathrm{C}\right)$ is the closed linear span of the exponential polynomials in the kernel. Furthermore any nonzero convolution operator on $H\left(\Sigma_{N} \mathrm{C}\right)$ is a surjective mapping.

Proof. This is a consequence of Theorems 1 and 2 together with the fact that $H\left(\Sigma_{N} \mathrm{C}\right)=H_{G}\left(\Sigma_{N} \mathrm{C}\right)$ (see Dineen [4]).

Now consider $E$ endowed with a locally convex topology and let $H(E)$ be the space of holomorphic functions on $E$ endowed with the compact open topology.

Proposition 4. Let $A$ be a convolution operator on $H_{G}\left(E, K_{c}\right)=H_{G}(E)$. Then the restriction of $A$ to $H(E)$ is convolution operator on $H(E)$.

Proof. $A=T *$ for some $T \in H_{G}^{\prime}(E)$. Let $C>0$ and $K$ a finite dimensional compact set such that $|T(f)| \leq C \sup _{z \in K}|f(z)|$ for all $f \in H_{G}(E)$.

(a) First we show that $T * f \in H(E)$ if $f \in H(E)$. Let $\omega_{0} \in E$. Then there exists a neighborhood of 0 in $E$, say $N$, such that $\sup _{z \in \omega_{0+K+N}}|f(z)|<+\infty$. Now if $\omega \in N$, then $\left|T * f\left(\omega+\omega_{0}\right)\right| \leq C \sup _{z} \in \omega_{0+K+N}|f(z)|$. Hence $T * f \in H(E)$.

(b) To complete the proof, we show that $T * f_{a} \rightarrow T * f$ whenever $f_{a} \rightarrow f$ in $H(E)$. Let $L$ be a compact set in $E$. Then $K+L$ is compact and hence $f_{a} \rightarrow f$ uniformly on $K+L$. Now if $l \in L$, then

$$
\begin{aligned}
\left|\left(T * f_{a}-T * f\right)(l)\right| & =\left|T\left(r_{-l}\right)\left(f_{a}-f\right)\right| \\
& \leq C \sup _{z \in K+L}\left|\left(f_{a}-f\right)(z)\right| .
\end{aligned}
$$

Hence $T * f_{\alpha} \rightarrow T * f$ uniformly on $L$ showing that $T *$ is a continuous mapping on $H(E)$.

\section{BIBLIOGRAPHY}

1. J. A. Barroso, Topologia em espaços de aplicações holomorfas entre espaços localmente convexos, An. Acad. Brasil. Ci. 43 (1971), 527-546.

2. P. J. Boland, Espaces ponderés de fonctions entières et de fonctions entières nucléaires sur un espace de Banach, C. R. Acad. Sci. Paris 75 (1972), 587-590.

3. - Some spaces of entire and nuclearly entire functions on a Banach space, J. Reine Angew. Math. (to appear in two parts).

4. S. Dineen, Holomorphic functions on locally convex topological vector spaces, Ann. Inst. Fourier (Grenoble), 23 (1973), 19-54. 
5. C. P. Gupta, Convolution operators and holomorphic mappings on a Banach space, Séminaire d'Analyse Moderne, no. 2, Université de Sherbrooke.

6. B. Malgrange, Existence et approximation des solutions des équations aux dérivées partielles et des équations des convolutions, Ann. Inst. Fourier (Grenoble) 6 (195556), 271-355. MR 19, 280.

7. Leopoldo Nachbin, Topology on spaces of holomorphic mappings, Ergebnisse der Math. und ihrer Grenzgebiete, Band 47, Springer-Verlag, New York, 1969. MR 40 \$7787.

8. B. A. Taylor, Some locally convex spaces of entire functions, Proc. Sympos. Pure Math., Vol. 11, Entire Functions and Related Parts Analysis, 1968.

DEPARTMENT OF MATHEMATICS, UNIVERSITY COLLEGE OF DUBLIN, BELFIELD, DUBLIN 4, IRELAND 